\title{
Electronic Patient Records in Sri Lankan Hospitals
}

\author{
Dr. Denham Pole MBBS, MD \\ Consultant to the Swiss Red Cross, Sri Lanka \\ E-mail address: drpole@gmail.com \\ URL : http://www.mdssrilanka.com
}

Sri Lanka Journal of Bio-Medical Informatics 2010;1(1):43-45

DOI: 10.4038/sljbmi.v1i1.1466

\begin{abstract}
Documentation places a heavy load on the staff of Sri Lankan government hospitals. Electronic patient documentation offers theoretical advantages, but attempts to computerize hospital records in the country have had only limited success. A project sponsored by the Austrian/Swiss Red Cross recently carried out in the Eastern Province has, however, been successful in computerizing the medical records of over 20 government hospitals. Local networks were set up in each hospital with workstations at the admission desk, in each ward and in some hospitals, in the ambulant out-patient departments. A basic clinical record is stored for retrieval when the patient returns. Costs were kept down by using the operating system LINUX and by local programming of the database. The main secret of success was continuous training of hospital staff over a 2 to 3 year period. The system is very much appreciated by the hospital staff as it makes their work more efficient and improves the quality of routine reports.
\end{abstract}

Keywords: ePR, Electronic Health Record, Hospital Information System, Healthcare Computing

\section{Background}

Sri Lanka enjoys universal health cover provided mainly by a country-wide system of hospitals financed, staffed and equipped by the Government. Its public health system, also run by the Government is one of the most comprehensive in the world with Medical Officers of Health maintaining community health in every rural and urban area. Such an extensive healthcare organization is heavily dependent on documentation starting at the out-patient and admission record and extending up to disease notification and national health statistics, not forgetting all the organizational and management records needed to exchange information on the way.

This documentation puts a heavy work-load on healthcare staff. As it is based almost entirely on handwritten paper records there is additional work in filing and retrieving information, in copying and summarizing it and in transmitting it from one department or institution to another. Many of the weaknesses of the healthcare system can be traced to lack of information that is stored somewhere but is not available where it is needed. But go to any matron's or medical records office in the country and you will see the heavy load of documentation work that is carried out by industrious and skilled healthcare staff.

A major revolution in information management of recent years has been the widespread use of computers to store and process information in many sectors including health. Electronic health records would seem to offer advantages over paper ${ }^{(1)}$ but in developing countries there are constraints to their full implementation. The main problems are lack of resources, limited computer skills and shortage of staff. A number of attempts to computerize Sri Lankan hospitals have failed because of this. To be successful, computer systems in developing countries will have low cost, be easy to use and will make routine work of limited staff more efficient. 
A project to introduce computerized records sponsored by the Austrian/Swiss Red Cross has recently been completed in the Eastern Province. In over 20 hospitals, clinical information is now stored in computers for all patients admitted in Batticaloa and Kalmunai Health District and in the Trincomalee General Hospital. The multi-disease surveillance software MDS ${ }^{(2)}$ was developed locally for the Ministry of Healthcare and Nutrition in collaboration with the World Health Organization using a revolutionary database system Caché ${ }^{(3)}$ that was initially developed for healthcare in the US.

Work started on this project in July 2006. The first small hospital (Chenkalady Rural Hospital) started using computerized records in February 2007 and the first large hospital (Batticaloa Teaching Hospital) in April 2007. Both of these hospitals are now recording outpatient and admission clinical details in the computer. At the end of 2009, 20 hospitals in the Eastern Province were using computers to store medical records. In one hospital (Trincomalee General Hospital) the computer system is also used to order diagnostic tests and display results and digital x-ray images can be seen on the screens in each ward.

In each hospital there is a computer installed at the front desk where patients are registered and another in each ward. In the wards the staff can see patient details and enter the diagnosis into the computer. This can then print useful reports such as the midnight census and patient discharge letters. Where the system is used for out-patients, each OPD doctor has a screen and keyboard on their desk and the electronic record has replaced paper. Administrative reports are also produced on computers connected to the network.

\section{Scope of the Electronic Patient Record}

In the design of the MDS software it was necessary to strike a delicate balance between enough information to make the stored patient record useful but not so complicated as to overwhelm the staff with too many details. A general principle adopted was to duplicate as far as possible the layout of the current manual records and to resist the temptation to "improve"on them.

Most input was made optional - a doctor who wants to enter the results of physical examinations can do so but others can switch this off. Booking the surgical theatre is hardly necessary for administrative staff so it can be removed to make their screens look less complicated.

In order to keep the input user-friendly we used pop-up lists for simple choices like religion, while longer lists such as residential villages were obtained from official sources and stored in the database. By combining the village list with the list of Public Health Offices it was possible for the computer to identify the correct office to notify a communicable disease from the patient's residential address. The list of drugs available in the pharmacy and the tests carried out in the hospital's laboratory or x-ray department could be added and updated by the corresponding department.

We faced a problem when codifying diseases. The ICD-10 coding, used by the Health Ministry for the quarterly health statistics report is normally made by specially trained staff in the medical records departments. Its use proved to be too complicated, however, for the nonstatistically trained hospital staff to use. Fortunately the alternative SNOMED (4) classification, used in the British National Health Service contains a link to ICD-10 and is quite clinically-friendly. The SNOMED clinical terms can therefore be entered by doctors and nurses (for example "Nucleotidase deficiency") and the computer finds the more 
administrative terms used in the ICD-10 (in this case "Disorders of plasma-protein metabolism, not elsewhere classified").

All this sophisticated programming carried out by Sri Lankans themselves was made possible by choosing a "web-based" approach to the screens where the only software needed on the workstation is an Internet browser (like "Firefox"). No data or other programs are needed so that if a ward computer fails it can be immediately replaced by a new one. The skills learnt in using the system can be used by the staff for Internet browsing and the opposite is also true. Use of the system becomes almost child's play and the screens give a feeling of familiarity.

As well as keeping the clinical record on individual patients, the software provides hospital directors with useful information to help run their hospitals like performance statistics and listing of admissions and discharges. And in every one of those 20 government hospitals it is the nurses and doctors who personally enter the medical data. The system contributes to public health by alerting the staff when there is a notifiable infection that must be notified to the Medical Officer of Health. It also produces the quarterly health statistics automatically.

\section{Conclusions}

While developing countries obviously have problems, computerization can transform many of these problems into opportunities. We have to be careful to avoid cost and complexity. The model used by MDS is a local area network using a separate server in each hospital but connected to other sites via the Internet. Hardware and development costs were kept down by using the highly efficient, easy to program Caché database and the LINUX operating system. This combination provided excellent performance on very low-cost machines and the webbased programming reduced travelling time needed for software maintenance.

The key to success however was training. During the 31/2 years of the project over 1000 staff were trained and the Red Cross local team provided continuous user support. It took most hospitals about one year before their skills were up to the level of running the systems themselves and outside support and regular training will always be necessary to ensure that the systems continue to function and that new staff are shown how to use the computer.

We were once told by Winston Churchill: You can fight an army, you can fight a nation, but you cannot fight an idea whose time has come. The computer's time has come and ePR will also come to a hospital near us sooner or later whether we like it or not. The MDS system has provided a good start to the computerization of hospitals in Sri Lanka, and it may even prove useful in other developing countries.

\section{References}

1. "Electronic Health Records", Harvard Public Health Review, Fall 2008

2. Available from http://www.mdssrilanka.com Accessed 15 December 2009

3. Available from http://www.intersystems.com/cache/index.html Accessed 15 December 2009

4. Available from http://www.connectingforhealth.nhs.uk/systemsandservices/data/snomed Accessed 15 December 2009 